\title{
Interface entre Teoría de la Carga Cognitiva y habilidades de lectura comprensiva en L2: Un estudio experimental mixto
}

\author{
Pedro Luis LUCHINI \\ Universidad Nacional de Mar del Plata \\ Facultad de Humanidades, Dpto. de Lenguas Modernas \\ UCAECE Mar del Plata \\ Facultad de Humanidades, Instituto de Lenguas \\ luchinipedroluis@gmail.com \\ Gabriela Mariel FERREIRO \\ Universidad Nacional de Mar del Plata \\ Facultad de Humanidades, Dpto. de Lenguas Modernas \\ ga16mafe@gmail.com
}

Recibido: marzo 2014

Aceptado: julio 2014

\section{RESUMEN}

Basada en la Teoría de la Carga Cognitiva (TCC), esta investigación busca explorar el grado en el que el Efecto Redundancia (EF) afecta las habilidades de lectura comprensiva en una L2 (segunda lengua) en un grupo de 24 alumnos, cuya lengua materna es el español, pertenecientes a una escuela privada en Mar del Plata, Argentina. Los participantes fueron divididos en dos grupos. Uno de los grupos fue expuesto a un único modo de instrucción: la lectura, mientras que para el otro grupo se recurrió a la integración de la lectura y la escucha. Los datos fueron analizados siguiendo un diseño experimental mixto, es decir una combinación de datos cualitativos con cuantitativos. Los resultados, que fueron sometidos a la técnica de triangulación, revelaron que los participantes que recibieron exposición a un solo modo de instrucción obtuvieron mejores resultados tras realizar una actividad de lectura comprensiva. Por último, y a la luz de estos hallazgos, se presentan algunas implicancias pedagógicas para la enseñanza de habilidades de lectura comprensiva en la clase de inglés.

Palabras clave: Lectura comprensiva, carga cognitiva, diseño de instrucción.

Interface between Cognitive Load Theory and L2 reading comprehension skills: A mixed designed experiment

\footnotetext{
ABSTRACT

The purpose of this research is to explore the extent to which the Redundancy Effect (RE) affects the L2 reading comprehension skills of a group of 24 young learners -whose L1 is Spanish- taking a low-intermediate course in English at a private school in Mar del Plata,
} 
Argentina. This group was divided into two sub-groups: A \& B. Sub-group A was exposed to a single mode of instruction (reading alone). Sub-group B was exposed to a multiple presentation mode (reading + listening). Data were gathered using a text that was carefully chosen to meet the students' age and English language proficiency level. After completing the reading task, both sub-groups filled out a questionnaire, and five students from each group were interviewed. Using a mixed research design, qualitative and quantitative information, coming from different instruments of data collection was cross-checked. Data triangulation was used to strengthen final results. Findings showed that sub-group A (reading alone) outperformed the other sub-group (reading + listening). Based on these results, some suggestions for the development of L2 reading comprehension skills were made, and some pedagogical implications were addressed.

Key words: Reading comprehension, cognitive load, instructional design.

\section{Interface entre la Théorie de la Charge Cognitive et les compétences en compréhension de la lecture L2: Une étude expérimentale mixte}

\section{RÉSUMÉ}

Basé sur la théorie de la charge cognitive, cette recherche vise à explorer la mesure dans laquelle l'effet de redondance affecte les compétences en lecture de compréhension dans une L2 (langue seconde) dans un groupe de 24 étudiants, dont la première langue est l'espagnol, l'appartenance à une école privée à Mar del Plata, en Argentine. Les participants ont été divisés en deux groupes. Un groupe a été exposé à un seul mode d'enseignement: lecture, tandis que pour l'autre groupe a été utilisé pour l'intégration de la lecture et de l'écoute. Les données ont été analysées selon un modèle expérimental mixte, c'est à dire une combinaison de données qualitatives avec quantitative. Les résultats, qui ont été soumis à la technique de triangulation, ont révélé que les participants qui ont reçu une exposition à un mode d'emploi simple surperformé après l'exécution d'une activité de compréhension de lecture. Enfin, à la lumière de ces résultats, des compétences pédagogiques pour l'enseignement de la compréhension en lecture en anglais implications de classe sont présentés.

Mots-clé: Compréhension à la lecture, la charge cognitive, la conception pédagogique.

SUMARIO: 1. Introducción. 2. Marco teórico. 3. Método. 3.1. Participantes. 3.2. Procedimiento para la recolección de datos e instrumentos. 4. Combinación de los datos: Triangulación de la información. 5. Resultados numéricos. 6. Resultados de valor. 6.1. Resultados de las encuestas. 6.2. Resultado de las entrevistas. 7. Discusión y conclusión. 8. Algunas implicancias metodológicas. 9. Limitaciones del estudio. 10. Investigaciones a futuro. 11. Bibliografía.

\section{INTRODUCCIÓN}

Una de las características más importantes en la adquisición de segundas lenguas (ASL) es la construcción de un sistema de conocimientos al que, eventualmente, se puede recurrir en forma automática al expresarse y comprender. Pese a saber que los procesos de automatización y reestructuración son centrales en 
las teorías cognitivas para ASL, aún no está claro qué estructuras deben ser automatizadas mediante la práctica y cuáles deben ser reestructuradas. Aquello que sabemos y usamos automáticamente puede no ser necesariamente aprendido mediante la construcción gradual de la automaticidad, sino que también puede estar basado en la interacción del conocimiento que ya poseemos (Cfr. Lightbown, Spada y White, 1993).

La importancia y dificultad para comprender textos es indiscutible. La comprensión no es un simple proceso cognitivo de acceso al significado de palabras y de combinación de las mismas. Implica mucho más que esto porque se requiere de la construcción de una representación mental (Kintsch, 1998; Zwaan y Radvansky, 1998). Por un lado, están los procesos lexicales para acceder al significado de palabras. Estos procesos recurren a la memoria para elaborar sobre el texto y realizar conexiones con el conocimiento que uno ya posee. Por el otro, los procesos de inferencia ayudan a integrar una determinada oración con otras y con conocimientos previos (Moss, Schunn, Schneider, McNamara y VanLehn, 2011). Dada esta vasta complejidad, la comprensión lectora se convierte en un importante predictor para el aprendizaje de una lengua debido a que comprende procesos mentales de aprendizaje, memoria y resolución de problemas.

La Teoría de la Carga Cognitiva (TCC) se centra en las relaciones existentes entre la memoria a corto y largo plazo y los efectos que surgen a partir de estas relaciones entre estos dos tipos de memoria en el aprendizaje y en la resolución de problemas (Yali, Chandler y Sweller, 2007). La TCC ha sido utilizada en el campo de la educación para generar técnicas y procedimientos de instrucción (Sweller, 2003, 2004). El Efecto Redundancia (ER) ocurre cuando la misma información es presentada a los alumnos mediante distintos modos de instrucción en forma simultánea (por ejemplo, de manera oral y escrita). Estas múltiples formas de presentación generan una carga cognitiva innecesaria que obstruye el aprendizaje (Chandler y Sweller, 1991; Sweller 2005; Sweller y Chandler, 1994).

Cuando se aprende a leer en una lengua extranjera, los estudiantes suelen ser provistos de una versión oral del texto escrito en forma concurrente. Frecuentemente, los docentes explícitamente sugieren que ambos modos de instrucción han de ser brindados simultáneamente para reforzar el aprendizaje. Pese a la popularidad de esta práctica áulica, su efectividad es altamente cuestionada porque, conforme a la TCC, la práctica de la lectura y escucha en forma simultánea resulta ser menos efectiva que la práctica de lectura solamente.

Esta investigación tiene como objeto explorar el grado en el que el ER afecta la habilidad de lectura comprensiva en dos grupos de jóvenes estudiantes de inglés en una escuela privada en Mar del Plata, Argentina. Durante el proceso de lectura comprensiva, uno de los grupos será expuesto a una modalidad de presentación múltiple (lectura y escucha), mientras que el otro solamente deberá leer un texto. En la primera parte de este trabajo se presentará la fundamentación teórica, luego 
se describirá el método utilizado (contexto, participantes e instrumentos para recolectar datos). En las secciones siguientes se detallarán los resultados y se discutirán los hallazgos. Finalmente, se brindarán algunas sugerencias con respecto a la interface entre la TCC y las habilidades de lectura comprensiva para la realización de futuras investigaciones.

\section{MARCO TEÓRICO}

En el campo de adquisición de segundas lenguas (L2s), la lectura comprensiva cumple un rol preponderante. Un número considerable de variables están involucradas en el proceso de la lectura comprensiva. En términos generales, cuando los estudiantes leen un texto recurren a varios procesos cognitivos para procesar información que incluyen la recuperación y almacenamiento de input nuevo. Para poder procesar esta información los estudiantes necesitan emplear estrategias de lectura que frecuentemente involucran procesos de comprensión que dan sentido a lo que leen.

La complejidad en los procesos de lectura comprensiva está dada principalmente por el resultado de las diferencias individuales de los estudiantes en el uso de estrategias para comprender textos, conjuntamente con lo que aprenden de esos textos (Moss, Schunn, Schneider y Mc Namara y VanLehn, 2011). Muchos procesos cognitivos subyacen a la comprensión lectora. Estos procesos están fundamentados en diferentes teorías que, por lo general, sugieren que el lector construye una situación modelo a modo de representación del contenido del texto que desvía la atención de la forma escrita de las oraciones que constituyen el texto e incorpora conocimiento que no se ciñe estrictamente al texto (Kintsch, 1988, 1998; McNamara y Magliano 2009; Zwaan y cols., 1995).

Un lector construye un modelo de situación por medio de un texto basado en las proposiciones contenidas en el propio texto, y trabaja sobre esta información utilizando el conocimiento previo a través de procesos de inferencia (Brantmeier, 2002; Kintsch, 1988, 1998; Zwaan, 1999; Zwaan y Radvansky, 1998). Estos procesos cognitivos frecuentemente comprenden una serie de técnicas como skimming (búsqueda de ideas principales), scanning (exploración), reconocimiento de familia de palabras, conjeturas, predicciones, activación de conocimiento general, realización de inferencias, separación de ideas principales de secundarias, entre otras (Barnet, 1988).

Es frecuente que los instructores utilicen un modo múltiple explícito al presentar textos para la enseñanza de lectura compresiva. Esto se debe a que creen que la provisión de textos en forma escrita y oral de modo simultáneo es realmente positiva a los efectos de realizar una lectura compresiva. Sin embargo, muchos estudios basados en la TCC sugieren que la presentación de información por múltiples canales resulta contraproducente para la comprensión. En efecto, se ha 
demostrado que leer y escuchar en forma simultánea es menos efectivo que leer solamente.

La TCC es una teoría para la instrucción que se desprende de la disciplina de la Ciencia Cognitiva, la cual tiene que ver con los procesos mentales del aprendizaje, memoria y resolución de problemas. Ésta describe las estructuras del aprendizaje con relación a un sistema de procesamiento de información que involucra la memoria a largo plazo, la cual almacena el conocimiento y habilidades en forma cuasi-permanente, y la memoria a corto plazo, que ejecuta actividades intelectuales asociadas con lo consciente (Sweller, 1999, 1994).

La información solo puede ser almacenada en la memoria a largo plazo tras haberle prestado atención y haber sido procesada por la memoria a corto plazo. Esta última, sin embargo, es limitada en cuanto a su capacidad y duración. La memoria a corto plazo es el medio que nos permite pensar en forma lógica y creativa, resolver problemas y ser expresivos. Este tipo de memoria está relacionada con el dónde y el cómo direccionamos nuestra atención al pensar en algo o al procesar información. La mayor limitación de la memoria a corto plazo es su capacidad para abordar no más de ocho elementos de información al mismo tiempo (Miller, 1956). Si la capacidad de esta memoria es superada durante el procesamiento de una determinada cantidad de información puede no ser recuperada. Estas restricciones en la memoria a corto plazo pueden, de algún modo, retrasar, obstaculizar y hasta impedir el aprendizaje.

Aprender consiste en la capacidad de codificar o almacenar conocimiento y habilidades en la memoria a largo plazo de tal modo que pueda ser fácilmente recuperada o aplicada de acuerdo con la necesidad. Este conocimiento es almacenado en una red de información estructurada, la cual está conectada con otras redes. Las redes pueden ser clasificadas en conceptos de alta y baja complejidad, dependiendo de la relevancia de la información que contengan. Estas redes de información jerárquica son conocidas como esquemas. Los esquemas integran el detalle y la complejidad a medida que un conocimiento más extensivo es adquirido en un área de contenido. Los esquemas que son aprendidos pueden ser recordados y aplicados con relativa facilidad. Por ejemplo, alguien que aprende a andar en bicicleta necesita concentrarse en el conocimiento y las habilidades necesarias para coordinar movimientos específicos. Después de varios años de andar en bicicleta, la mayor parte de la gente puede hacerlo casi automáticamente. A medida que la automatización se desarrolla, hay una reducción en la necesidad de la concentración o una disminución en la carga cognitiva. Por consiguiente, podemos afirmar que cuando algo ha sido aprendido, ha sido codificado efectivamente en la memoria a largo plazo y puede luego ser recuperado en caso de necesidad.

Se insiste en la idea de que los recursos de la memoria a corto plazo son limitados en tanto que tan solo unos pocos elementos de información pueden ser 
considerados en un determinado momento. La información nueva, que es cargada con un elemento de interactividad de alto nivel, impone en los estudiantes una carga cognitiva mayor a la impuesta por los mismos elementos debido a que se crea la necesidad de considerar las relaciones entre estos elementos. Por consiguiente, un alto nivel de material interactivo aumenta las dificultades que resultan de las limitaciones de la memoria a corto plazo.

En contextos áulicos se debe tener en cuenta que la calidad del diseño instruccional puede afectar a la enseñanza de la lectura comprensiva en L2 y la adquisición en general. Esto significa que si el diseño instruccional recibiera mayor consideración con relación al rol y las limitaciones de la memoria a corto plazo para la adquisición del lenguaje, podrían obtenerse mejores resultados. Desde su concepción a principios de los 80, la TCC ha sido utilizada para desarrollar variadas estrategias de instrucción que han demostrado empíricamente ser superiores a las utilizadas convencionalmente (Sweller, 1999).

Existen dos tipos de carga cognitiva: carga cognitiva intrínseca y extrínseca. La primera se debe exclusivamente a la naturaleza intrínseca o dificultad propia de un contenido para ser aprendido. La carga cognitiva intrínseca no puede ser adaptada por un diseño instruccional. Por ejemplo, un contenido que es complejo en interactividad de elementos permanece complejo, independientemente del modo en que sea presentado. Por otra parte, la carga cognitiva extrínseca o externa se debe al tipo de diseño de los materiales de instrucción utilizados para presentar información a los estudiantes. Al alterar el diseño de estos materiales didácticos, el nivel de carga cognitiva extrínseca varía, facilitando o complejizando el proceso de aprendizaje. En otras palabras, el rediseño de los materiales para la instrucción puede reducir o aumentar los niveles de carga cognitiva externa y de este modo mejorar u obstaculizar el aprendizaje (Cooper, 1998). Un diseño instruccional recomendable reduce la carga cognitiva externa o innecesaria, aumentando el esquema de construcción y la automatización. La TCC ha sido utilizada en gran parte para elaborar procedimientos de instrucción (Sweller, 2003, 2004). Uno de estos procedimientos es el ER.

El EF ocurre cuando la misma información es presentada a los alumnos por diferentes canales. La presentación de información utilizando un formato múltiple obliga a los alumnos a sincronizar psicológicamente esas variadas formas. El tener que procesar la misma información en múltiples formas impone una carga cognitiva externa en los estudiantes que dificulta el aprendizaje (Chandler y Sweller, 1991; Sweller, 2005; Sweller y Chandler, 1994). Y el ER ocurre cuando el mismo texto es presentado en forma oral y escrita simultáneamente (Craig, Gholson y Driscoll, 2002; Kalyuga, Chandler y Sweller, 2000, 2004; Mayer, Heiser y Lonn, 2001). La combinación de dos modalidades de presentación de un mismo texto (oral y escrito) realizadas al unísono interfiere notablemente con la 
comprensión lectora de los alumnos más que si se utilizara una modalidad simple, como lo es la lectura solamente.

Frecuentemente en las clases de inglés como segunda lengua o lengua extranjera, los docentes, expresamente, asumen que un texto debe ser presentado a sus alumnos tanto en forma oral como escrita. Esta creencia promueve el uso de múltiples recursos para la instrucción con el objetivo de proveer a los estudiantes de un conocimiento lingüístico más enriquecedor (Yali, Chandler y Sweller, 2007). Cuando el objetivo central es enseñar a leer en forma crítica, es una tendencia entre los docentes de lenguas extranjeras, proveer a los alumnos de materiales que ofrecen múltiples modalidades para que ellos los procesen. Detrás de esta práctica de uso frecuente, existe una creencia desacertada. Se cree que la integración sincrónica en los modos de presentación favorece el aprendizaje.

La TCC sugiere que el diseño instruccional que exige a los alumnos distribuir su atención entre múltiples fuentes de información (por ejemplo: lectura y escucha) es ineficaz para el aprendizaje. Se desprende entonces que la información debe ser presentada por canales que no imponen fuertes cargas cognitivas externas (por ejemplo: solo lectura). Las presentaciones de carácter múltiple no se ajustan a este criterio. Por consiguiente, si el objetivo es enseñar a los alumnos a leer en forma efectiva, los canales de información han de ser fusionados en uno para ahorrar a los estudiantes la realización de integraciones mentales innecesarias que interfieren con el aprendizaje.

En situaciones en las que el modo de instrucción demanda un alto nivel de inteligibilidad/comprensibilidad, se debe utilizar una única forma de instrucción. Para facilitar el proceso de comprensión se debe omitir todo aquel material de instrucción que sea redundante. La habilidad lectora por sí sola demanda la utilización de un alto nivel cognitivo. Por lo tanto, si el material escrito se presenta en conjunto con la escucha del mismo texto en forma simultánea, la comprensión se verá obstaculizada.

\section{MÉTODO}

\subsection{Participantes}

Participaron en esta investigación 24 alumnos de 13 años, inscriptos en un curso de nivel pre-intermedio en el CADS (Colegio Atlántico del Sur), Mar del Plata, Argentina. CADS es una escuela privada en la cual los alumnos reciben 4 horas semanales de instrucción en inglés, 2 veces a la semana. Los alumnos aprenden inglés desde el jardín de infantes hasta concluir con los estudios secundarios. Cuando los datos fueron recopilados estos alumnos se encontraban cursando su primer año en la secundaria. En este curso, objeto de estudio de esta investigación, los alumnos son preparados para rendir el examen estandarizado PET Cambridge Exam, ofrecido por Cambridge Local Syndicate. Los participantes fueron seleccionados al azar en grupo A y B. El grupo A recibió exposición a un único 
modo de instrucción (solo lectura). El grupo B debió leer y escuchar el contenido del mismo texto en forma simultánea.

\subsection{Procedimiento para la recolección de datos e instrumentos}

Para la recopilación de datos se emplearon tres tipos de instrumentos. Por un lado, se utilizó un texto de aproximadamente 500 palabras. El mismo fue seleccionado deliberadamente del libro de curso de los alumnos PET Result (Baker, 2001) para garantizar que su complejidad lingüística y extensión no se convirtieran en factores internos que -eventualmente - pusieran en peligro la validez de la investigación.

Tres jueces utilizaron el método inter-evaluador para analizar los datos recolectados. Segmentaron el texto en ideas principales y secundarias. Inicialmente, dos de los evaluadores trabajaron en forma conjunta para identificar las ideas principales. Posteriormente, el tercer evaluador confrontó lo realizado por los otros dos para homogenizar los resultados. En los casos que hubo discrepancias, los tres evaluadores discutieron lo pertinente hasta alcanzar un consenso. Se identificó un total de nueve ideas principales. Las mismas fueron utilizadas como respuesta patrón para analizar los resúmenes de los alumnos y evaluar sus producciones. La medición de las ideas principales en cada grupo resultó del número total de ideas principales divididas por 108 ideas principales (12 alumnos en cada grupo x 9 ideas principales: respuesta patrón).

El texto fue fragmentado en cinco párrafos, cada uno de ellos similar en longitud (aproximadamente 100 palabras). Estos párrafos fueron mostrados a los alumnos en cinco diapositivas de power point. Cada diapositiva fue proyectada por unos 30 segundos aproximadamente. Los alumnos no controlaron el ritmo del cambio de las diapositivas. El tiempo asignado para que los alumnos leyeran cada diapositiva fue estimado considerando una experiencia piloto realizada por el docente a cargo del grupo previa a la recolección de datos. Dicha experiencia posibilitó calcular el tiempo promedio que llevó a los alumnos leer y comprender extractos de una complejidad y extensión similar.

Tal como fuera expresado anteriormente, se le solicitó al Grupo A leer la narración de las diapositivas, mientras que el Grupo B, además de acceder a las diapositivas escuchó un audio en forma simultánea sobre la misma narración. La presentación de diapositivas fue mostrada a ambos grupos individualmente en dos turnos consecutivos. Inmediatamente después de completar las actividades de lectura y escucha, los alumnos debieron escribir un resumen de lo que habían leído, incluyendo tanta información como pudieran recordar. Se les permitió escribir sus resúmenes tanto en español como en inglés conforme a sus preferencias con el propósito de facilitarles la expresión de ideas. El Gráfico 1 debajo ilustra la primera diapositiva que fuera mostrada a los alumnos. 


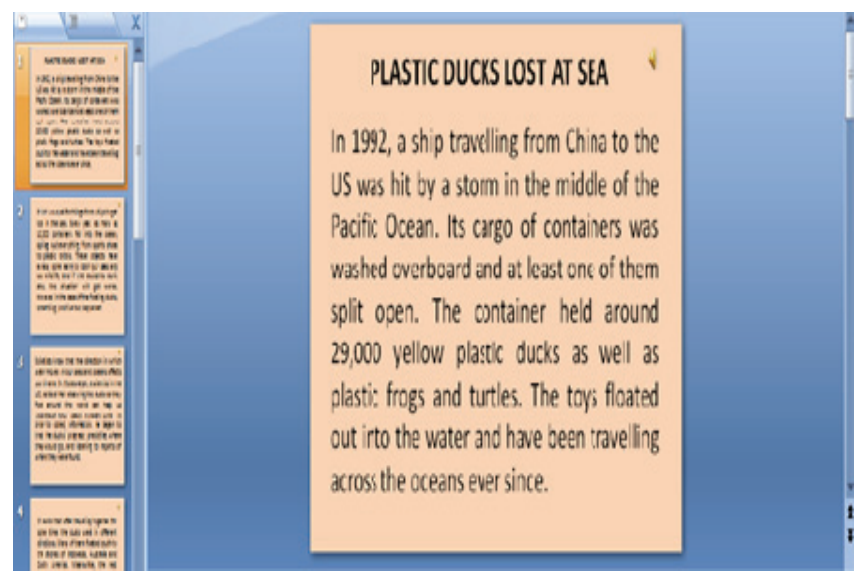

Gráfico 1: Primera diapositiva presentada a los alumnos en ambos grupos

Por otro lado, y con el objeto de indagar sobre la experiencia realizada y obtener, de este modo, las opiniones y percepciones de los dos grupos con respecto a las dos modalidades de presentación utilizadas (grupo A: simple, grupo B: múltiple), inmediatamente después de entregar sus resúmenes, los participantes, en ambos grupos, completaron una encuesta cerrada. Este segundo instrumento consistió en un listado de cinco preguntas. En cada pregunta se solicitó a los participantes exteriorizar su reacción eligiendo uno de los puntos de escala. Este instrumento fue diseñado especialmente para esta investigación dado que no existe un instrumento con estas características preparado para este fin. Las preguntas utilizadas se detallan a continuación:

1- Nivel de comprensión del texto: ¿Te costó mucho trabajo comprender el texto?

2- Nivel de comprensión del vocabulario:

a- ¿Encontraste muchas palabras en el texto que desconocías?

b- ¿En qué medida estas palabras nuevas influenciaron en la compresión del texto?

3- Longitud del texto: ¿En qué medida la longitud del texto influyó en su comprensión?

4- ¿En qué medida el tema del texto influyó en su comprensión?

5- ¿Preferís un texto sin escucharlo o leerlo y escucharlo al mismo tiempo?

De la pregunta número 1 a la 4 se empleó la siguiente banda de escala: 


\begin{tabular}{|l|l|l|}
\hline alto/mucho & medio/regular & bajo/poco \\
\hline
\end{tabular}

Asimismo, la pregunta número 5 solo presentó dos opciones:

\begin{tabular}{|l|c|}
\hline Lectura y escucha & Lectura \\
\hline
\end{tabular}

El tercer instrumento consistió en una entrevista en profundidad semiestructurada. Se eligieron 5 participantes de cada grupo al azar para ser entrevistados. Las entrevistas fueron grabadas y luego transcriptas para su posterior análisis e interpretación. El tipo de preguntas realizadas en las entrevistas fueron consistentes con las realizadas en la encuesta. Esto se hizo con el objetivo de validar los datos obtenidos en las encuestas.

\section{COMBINACIÓN DE LOS DATOS: TRIANGULACIÓN DE LA INFORMACIÓN}

En función de los beneficios aportados por el diseño de evaluación mixto, en este trabajo se combinarán datos cualitativos con cuantitativos entre sí. Este tipo de diseño permite identificar y explicar resultados contradictorios o ambiguos y verificar fehacientemente las interpretaciones finales (Lynch, 1996). La combinación de datos permite una mejor comprensión de los problemas de investigación. En este estudio se utilizó la técnica de triangulación de la información de manera tal que los datos numéricos (resultado experimental) pueden ser comparados y contrastados con los hallazgos provenientes de métodos cualitativos (encuestas y entrevistas).

Denzin (1989) define a la triangulación como la aplicación y combinación de varias metodologías de la investigación en el estudio de un mismo fenómeno y establece diferentes maneras de utilizar los procedimientos de triangulación, organizando posibilidades y alternativas para adoptar la que sea más adecuada al estudio en cuestión. La información proveniente de múltiples fuentes permite ser comparada para verificar si se hallan resultados similares. Este proceso contribuye a validar los resultados finales.

\section{RESULTADOS NUMÉRICOS}

Tras haber expuesto a ambos grupos a diferentes formas de presentación, se observaron diferencias significativas en los resultados. Los alumnos que únicamente leyeron el texto identificaron 51 ideas principales sobre un total de 108 recolectadas por ambos grupos. Los alumnos que recibieron exposición conforme al modo dual de instrucción solo reconocieron 32 ideas principales. La Tabla 1, a 
continuación, muestra estos resultados, tomando en cuenta el número de ideas principales identificadas por alumno en cada grupo y por grupo en su totalidad.

\begin{tabular}{|c|c|c|c|}
\hline \multicolumn{2}{|c|}{ Grupo A: Solo lectura } & \multicolumn{2}{|c|}{$\begin{array}{l}\text { Grupo B: Lectura y } \\
\text { escucha }\end{array}$} \\
\hline Participantes & $\begin{array}{l}\text { Ideas } \\
\text { principales }\end{array}$ & Participantes & $\begin{array}{l}\text { Ideas } \\
\text { principales }\end{array}$ \\
\hline Alumno 1 & 6 & Alumno 1 & 6 \\
\hline Alumno 2 & 2 & Alumno 2 & 6 \\
\hline Alumno 3 & 6 & Alumno 3 & 1 \\
\hline Alumno 4 & 4 & Alumno 4 & 5 \\
\hline Alumno 5 & 4 & Alumno 5 & 2 \\
\hline Alumno 6 & 4 & Alumno 6 & 4 \\
\hline Alumno 7 & 3 & Alumno 7 & 0 \\
\hline Alumno 8 & 7 & Alumno 8 & 0 \\
\hline Alumno 9 & 6 & Alumno 9 & 2 \\
\hline Alumno 10 & 5 & Alumno 10 & 1 \\
\hline Alumno 11 & 2 & Alumno 11 & 3 \\
\hline Alumno 12 & 2 & Alumno 12 & 2 \\
\hline TOTAL & 51 & TOTAL & 32 \\
\hline
\end{tabular}

Tabla 1: Número de ideas principales identificadas por alumno en ambos grupos

El análisis de los resultados indicó que aquellos alumnos que fueron expuestos a la modalidad de solo lectura obtuvieron mejores resultados que los alumnos del Grupo B, expuestos a la modalidad de instrucción con redundancia. Así lo demuestran los resultados consignados en Tabla 2, donde se indican las medias para cada grupo y el puntaje de diferencia de medias. Esta diferencia sugiere, en términos generales, que el Grupo A reconoció más ideas principales que el Grupo B.

\begin{tabular}{|c|c|c|}
\hline Grupo A & $47.22 \%$ & \\
\cline { 1 - 2 } Grupo B & $29.62 \%$ & $\mathbf{1 7 . 6 \%}$ \\
\hline
\end{tabular}

Tabla 2: Porcentajes de cada grupo y puntaje de diferencia

Estos datos confirman nuestra hipótesis inicial en la que expresamos que un único modo de instrucción reduce el ER y, por consiguiente, promueve la utilización de estrategias que tienden a optimizar la lectura comprensiva. 
Recordamos una vez más que un diseño de instrucción que integra un modo múltiple impone una carga cognitiva externa que obstruye la comprensión lectora.

\section{RESULTADOS DE VALOR}

\subsection{Resultados de las encuestas}

Para cada una de las cinco preguntas, los alumnos identificaron con una tilde sus preferencias. A continuación se muestran las preguntas seguidas del detalle de resultados obtenidos para cada una, sobre un total de 12 alumnos en cada grupo:

1- Nivel de comprensión del texto: ¿Te costó mucho trabajo comprender el texto?

\begin{tabular}{|l|l|l|l|}
\hline Grupos & alto/mucho & medio/regular & bajo/poco \\
\hline Lectura y escucha & 1 & 5 & 6 \\
\hline Lectura & ninguno & 3 & 9 \\
\hline
\end{tabular}

2- Nivel de comprensión del vocabulario:

a) ¿Encontraste muchas palabras en el texto que desconocías?

\begin{tabular}{|l|l|l|l|}
\hline Grupo & alto/ mucho & medio/regular & bajo/poco \\
\hline Lectura y escucha & ninguno & 7 & 5 \\
\hline Lectura & ninguno & 4 & 8 \\
\hline
\end{tabular}

b) ¿En qué medida estas palabras nuevas influenciaron en la comprensión del texto?

\begin{tabular}{|l|l|l|l|}
\hline Grupo & alto/mucho & medio/regular & bajo/poco \\
\hline Lectura y escucha & 2 & 5 & 5 \\
\hline Lectura & ninguno & 3 & 9 \\
\hline
\end{tabular}

3-Extensión del texto: ¿En qué medida la extensión del texto influyó en su comprensión?

\begin{tabular}{|l|l|l|l|}
\hline Grupo & alto/ mucho & medio/regular & bajo/poco \\
\hline Lectura y escucha & ninguno & 5 & 7 \\
\hline Lectura & 1 & 3 & 8 \\
\hline
\end{tabular}

4-¿En qué medida el tema del texto influyó en su comprensión?

\begin{tabular}{|l|l|l|l|}
\hline Grupo & alto/ mucho & medio/regular & bajo/poco \\
\hline
\end{tabular}




\begin{tabular}{|l|l|l|l|}
\hline Lectura y escucha & 2 & 4 & 6 \\
\hline Lectura & ninguno & 2 & 10 \\
\hline
\end{tabular}

5- ¿Preferís leer un texto sin escucharlo o leerlo y escucharlo al mismo tiempo?

\begin{tabular}{|l|l|l|}
\hline Grupo & Lectura & Lectura y escucha \\
\hline Lectura y escucha & 7 & 5 \\
\hline Lectura & 6 & 6 \\
\hline
\end{tabular}

\subsection{Resultado de las entrevistas}

Se recuerda que los datos consignados en esta sección corresponden a las entrevistas semi-estructuradas realizadas a 5 alumnos de cada grupo. Estos alumnos fueron seleccionados al azar. A continuación se detallan las preguntas realizadas y las respuestas consignadas en palabras y números. Los números representan la cantidad de alumnos en cada grupo que respondió esa pregunta.

1- ¿Comprendiste la totalidad del texto?

$(\ldots)=$ cantidad de alumnos; 5 alumnos $\mathrm{x}$ columna -10 en total.

\begin{tabular}{|l|l|}
\hline Grupo: Lectura y Escucha & \multicolumn{1}{|c|}{ Grupo: Lectura } \\
\hline Todo (2) & Todo (5) \\
\cline { 1 - 1 } Casi todo (1) al principio fue más difícil & \\
\cline { 1 - 1 } Algo (1) algunas palabras costaron & \\
\cline { 1 - 1 } $\begin{array}{l}\text { Poco (1) el principio y final sí; el medio } \\
\text { no. }\end{array}$ & \\
\hline
\end{tabular}

2- Si fue así, ¿cómo comprendiste el texto?

\begin{tabular}{|l|l|}
\hline \multicolumn{1}{|c|}{ Grupo: Lectura y Escucha } & \multicolumn{1}{c|}{ Grupo: Lectura } \\
\hline No respondieron (4) & No respondieron (2) \\
\hline $\begin{array}{l}\text { Leo antes del audio y luego escucho y } \\
\text { leo (1) }\end{array}$ & $\begin{array}{l}\text { La segunda lectura me ayudó a } \\
\text { comprender (2) }\end{array}$ \\
\hline & $\begin{array}{l}\text { Hace muchos años q estudio y me } \\
\text { encanta (1) }\end{array}$ \\
\hline
\end{tabular}

3- ¿Qué fue lo que te ayudó a comprenderlo?

\begin{tabular}{|l|l|}
\hline \multicolumn{1}{|c|}{ Grupo: Lectura y Escucha } & \multicolumn{1}{c|}{ Grupo: Lectura } \\
\hline $\begin{array}{l}\text { Me ayudó el audio, la pronunciación de } \\
\text { las palabras (1) }\end{array}$ & La segunda lectura (3) \\
\hline Las palabras conocidas: las relacionaba & Hace muchos años que estudio y me \\
\hline
\end{tabular}




\begin{tabular}{|l|l|}
\hline con las que desconocía (1) & encanta (1) \\
\hline El audio me ayudó a comprender (2) & $\begin{array}{l}\text { Mientras leía imaginaba la situación } \\
\text { para recordarla, relacionaba hechos (1) }\end{array}$ \\
\hline La lectura, no el escuchar el texto (1) & \\
\hline
\end{tabular}

4- ¿Cómo identificaste las ideas principales?

\begin{tabular}{|l|l|}
\hline \multicolumn{1}{|c|}{ Grupo: Lectura y Escucha } & \multicolumn{1}{c|}{ Grupo: Lectura } \\
\hline No supo responder (3) & $\begin{array}{l}\text { Presto atención al título, cada vez que } \\
\text { aparecía la palabra estaba atento. Salteo } \\
\text { ejemplos para re-leer lo importante (1) }\end{array}$ \\
\hline $\begin{array}{l}\text { Cada párrafo tiene su contenido, su } \\
\text { forma, su estructura, su tema (1) }\end{array}$ & $\begin{array}{l}\text { No supo explicarlo pero citó ejemplos } \\
\text { que claramente diferenciaron ideas } \\
\text { principales de secundarias (1) }\end{array}$ \\
\hline $\begin{array}{l}\text { Presto atención a la repetición de } \\
\text { palabras (las ideas principales las fui } \\
\text { viendo al hacer el resumen) (1) }\end{array}$ & $\begin{array}{l}\text { Presto atención al título, las primeras } \\
\text { oraciones de cada párrafo me ayudaron } \\
\text { a comprender el resto. (1) }\end{array}$ \\
\hline & Según la importancia de las ideas (1) \\
\hline & No supo responder (1) \\
\hline
\end{tabular}

5- ¿Encontraste muchas palabras nuevas en el texto que te dificultaron la comprensión del texto? Cuáles, por ejemplo? ¿Te acordás de alguna en particular?

\begin{tabular}{|l|l|}
\hline \multicolumn{1}{|c|}{ Grupo: Lectura y Escucha } & \multicolumn{1}{c|}{ Grupo: Lectura } \\
\hline Al principio entorpecieron, luego no (1) & $\begin{array}{l}\text { Las palabras nuevas no afectaron la } \\
\text { comprensión (1) }\end{array}$ \\
\hline No (1) & Algunas palabras nuevas: cargo (1) \\
\hline $\begin{array}{l}\text { Desconocía algunas palabras, no } \\
\text { recordaba el significado, pero las sabía } \\
(2)\end{array}$ & $\begin{array}{l}\text { No muchas pero las relaciono y las } \\
\text { entiendo (1) }\end{array}$ \\
\hline $\begin{array}{l}\text { No muchas: board, overboard, por la } \\
\text { borda (1) }\end{array}$ & $\begin{array}{l}\text { Las palabras nuevas, a otras no las } \\
\text { recordaba; me ayudó el contexto, las } \\
\text { relacionaba (1) }\end{array}$ \\
\hline & Hubiese preferido leer y escuchar (1) \\
\hline
\end{tabular}

6- En actividades de lectura comprensiva como esta ¿preferís leer el texto o leerlo y escucharlo simultáneamente? ¿Por qué?

\begin{tabular}{|l|l|}
\hline \multicolumn{1}{|c|}{ Grupo: Lectura y Escucha } & \multicolumn{1}{c|}{ Grupo: Lectura } \\
\hline Leer (3) no tiene el mismo ritmo de su & Leer (5) es más fácil, en silencio mejor. \\
lectura el audio; me cuesta prestar & Pude comprender más. Pude manejar \\
\hline
\end{tabular}




\begin{tabular}{|l|l|}
\hline atención a las dos cosas. & $\begin{array}{l}\text { los tiempos de lectura y volver atrás } \\
\text { cuando era necesario. Seguir a la } \\
\text { persona que lee me distrae y no puedo } \\
\text { volver atrás para comprender; si hago } \\
\text { ambas cosas mezclo todo. }\end{array}$ \\
\hline $\begin{array}{l}\text { Generalmente prefiero leer, pero esta } \\
\text { vez el audio me ayudó (1) }\end{array}$ & \\
\hline $\begin{array}{l}\text { En inglés prefiero leer. En Español } \\
\text { prefiero escuchar (1) }\end{array}$ & \\
\hline
\end{tabular}

En la próxima sección se discutirán estos resultados teniendo en cuenta la información proveniente de los diferentes instrumentos de recolección de datos utilizados en el estudio.

\section{DISCUSIÓN Y CONCLUSIÓN}

Según demuestran los datos en su faz experimental y de valor, la hipótesis inicial se confirma. El grupo A, expuesto solo a la lectura, superó los resultados obtenidos por el otro grupo. Un diseño instruccional que integra un modo de exposición múltiple impone una carga cognitiva externa que afecta a la comprensión lectora. Este concepto suele contradecir algunas teorías de la adquisición de segundas lenguas, y sobre todo las prácticas docentes, porque en muchos contextos se promulga la utilización de presentaciones múltiples para desarrollar las habilidades de lectura comprensiva.

Como ya se explicó, existe una falsa creencia sustentada en el hecho de que cuánta más información se brinde a los alumnos, y esto implica también apelar a múltiples canales para la recepción de esta información, el aprendizaje será más efectivo. Sin embargo, los datos demuestran que cuando un mismo texto es presentado usando modalidades múltiples ocurre lo contrario.

La decodificación de un texto en una L2 (segunda lengua) utilizando un modo único de instrucción de por sí implica una carga cognitiva severa sobre la memoria activa. Por lo tanto, es improbable que estudiantes de una L2 tengan suficiente capacidad de memoria activa para poder manipular un modo de instrucción múltiple que exige realizar la lectura y escucha en forma simultánea.

Investigaciones realizadas sobre TCC ofrecen respuestas para controlar la carga intrínseca cognitiva alta por medio de enfoques que consideran el conocimiento previo de los estudiantes. Sin embargo, algunas tareas imponen desafíos mayores que otras, porque generan un alto nivel de interactividad. Y justamente una de estas actividades desafiantes es la comprensión de la lectura de una lengua extranjera porque requiere la construcción de representaciones mentales complejas.

La TCC argumenta que la carga dirigida hacia la construcción, procesamiento y mecanización de esquemas puede ser manipulada y optimizada por medio de 
diseños efectivos de instrucción de manera que favorecen el aprendizaje, dirigiendo la atención hacia los procesos de aprendizaje más relevantes. Los hallazgos de la presente investigación muestran que este resultado fue alcanzado en forma más efectiva por el grupo A, expuesto a una modalidad de presentación simple.

No obstante, a la luz de los datos de valor provenientes de la encuesta con respecto al grupo $\mathrm{A}$, vale la pena destacar una serie de inquietudes que merecen ser discutidas. Con respecto al grupo A, solo lectura, en la pregunta N2 de la encuesta, los alumnos destacaron la poca interferencia de palabras nuevas que pudieron haber obstaculizado la comprensión del texto. Se sabe que la falta de gramática y vocabulario afecta tanto a la producción como a la percepción en L2. En un estudio en el que oyentes evaluadores escucharon y analizaron muestras de habla para determinar niveles de comprensibilidad, Trofimovich e Isaac (2012) descubrieron que los errores gramaticales y algunos aspectos del vocabulario en L2 afectan y desvirtúan la atención de los oyentes evaluadores, afectando su comprensibilidad.

Varonis y Gass (1982), por su parte, descubrieron que las oraciones agramaticales afectan a la comprensibilidad. Fayer y Krasinski (1987) y Varonis y Gass (1982) sostienen que los errores gramaticales y de vocabulario afectan negativamente a la comprensibilidad. En un estudio experimental, Munro y Derwing (1999) indican que oyentes evaluadores señalaron que los errores de gramática y vocabulario atentaron significativamente contra la inteligibilidad y comprensibilidad. Estos datos se correlacionan, en parte, con los resultados de este estudio. Los alumnos del grupo A indicaron no tener problemas con la gramática y el vocabulario del texto al que fueron expuestos, por lo tanto se infiere que pudo haber sido ésta la razón por la cual lograron identificar más ideas principales, y tener un mejor nivel de comprensión del texto. Posiblemente, fue esto lo que los llevó a conseguir puntajes más altos que los del otro grupo. Estos datos son coincidentes con los comentarios que estos mismos alumnos hicieron en las entrevistas (Cfr. Pregunta 1, entrevista), donde la mayoría (5 alumnos) afirmó que no tuvo dificultad con la complejidad del texto.

En la pregunta N3 de la encuesta se hace referencia a la extensión del texto empleado. Con respecto a este tema, ambos grupos manifestaron no haber tenido inconvenientes. Sabemos que estos alumnos son capacitados en el desarrollo de las cuatro macro-habilidades lingüísticas para dar un examen internacional que requiere, entre otras cosas, la destreza de leer textos similares al empleado en este estudio, y completar tareas equivalentes a la realizada aquí, todo esto en un tiempo acotado.

Son notorios los puntajes altos asignados a la pregunta N4, donde los participantes en ambos grupos señalan no haber tenido problemas con la temática del texto. Es necesario recordar que los alumnos no habían visto el texto seleccionado antes de realizar este experimento. Sin embargo, es importante recordar que estos alumnos están familiarizados con este tipo de textos, porque los 
utilizan en como parte de la preparación a la que están diariamente expuestos para dar el examen internacional. Tal vez, fue esta preparación específica lo que explica, en parte, por qué los dos grupos obtuvieron resultados altos en estas dos respuestas (N3 y N4) de la encuesta.

Otro puntaje del grupo A que también llama la atención es el asignado a la preferencia de modalidades de presentación. Se observa una discrepancia entre los datos numéricos y los juicios de valor que estos alumnos hacen con respecto a su elección en la encuesta. En casos como éste, donde las estrategias metodológicas utilizadas arrojan hallazgos disímiles, la triangulación de los datos ofrece una oportunidad para que se elabore una perspectiva más amplia en cuanto a la interpretación del fenómeno analizado, porque señala su complejidad, y esto a su vez enriquece el estudio y brinda la oportunidad de que se realicen nuevos planteamientos.

Por un lado, los números indican que los alumnos que fueron expuestos al tratamiento lectura solamente, obtuvieron mejores puntajes que los otros. Por el otro, el $50 \%$ de los alumnos encuestados en este mismo grupo admite que prefieren una modalidad dual de instrucción. Conforme a la hipótesis planteada en este trabajo se puede inferir que la opinión dividida en este grupo tiene que ver con la creencia, aparentemente compartida con los docentes y equivocada, de que el leer y escuchar información al mismo tiempo refuerza la comprensión lectora. Otra posible interpretación podría llevar a la conclusión de que estos resultados cualitativos tan solo reflejan el deseo, por parte de este grupo, de realizar esta misma experiencia de un modo distinto. Por otra parte, en vista de los datos reunidos por el grupo B para esta misma pregunta, esta última interpretación también podría considerarse válida.

Es interesante resaltar en los dos grupos la falta de comentarios con respecto a cómo comprendieron el texto. Existen varias razones que pueden dar sustento a esta reacción por parte de los alumnos. Por un lado, está la dificultad de reflexionar, racionalizar y poner en palabras lo realizado en una tarea que demanda un alto proceso cognitivo. En casos como éstos se apela a la metacognición. Es decir, al conocimiento que uno tiene acerca de sus propios procesos cognitivos $\mathrm{y}$, por otro, con la supervisión activa y constante regulación y organización de estos procesos en relación con los objetos o datos cognitivos sobre los que actúan, regularmente en función de un objetivo concreto. La metacognición se pone en práctica cuando se tiene conciencia de la mayor dificultad para completar una tarea que otra (White y Rant, 2002; Lam, 2009; Lightbown, 2000), como lo demuestra esta encuesta/entrevista, donde se espera que los alumnos reflexionen sobre sus propios procesos de aprendizaje. Por otro lado, está la falta de experiencia de estos alumnos, o la carencia de sensibilización lingüística acerca del enfoque utilizado con relación al material de aprendizaje. 
Haciendo un recorrido por las respuestas tanto de las encuestas como de las entrevistas se observa un interesante escenario en el que aparecen las estrategias de aprendizaje, y en particular las de lectura comprensiva (Cfr. Entrevista: "La 2da lectura", "Presto atención al título, cada vez que aparecía la palabra estaba atento", "Salteo ejemplos para re-leer lo importante"), y las de adquisición de vocabulario (Cfr. Entrevista: "Las palabras nuevas, a otras no las recordaba; me ayudo por el contexto, las relaciono").

Las estrategias de aprendizaje configuran y organizan los elementos estructurales relacionados con formas superiores de actividad cognitiva que intervienen en la producción y organización de la información, y que conforman la infraestructura cognitiva. Por otro lado, aparecen los estilos de aprendizaje, y aquí debemos hacer referencia a los diferentes canales involucrados en este proceso. Estos estilos describen la forma en que cada individuo aprende. Son la clave para comprender las diferentes preferencias de los alumnos durante su proceso de aprendizaje. Podemos entenderlos desde cómo una persona percibe y produce conocimientos mediante tres estilos o canales diferentes: visual, auditivo y kinestésico. Por ejemplo, cuando un individuo logra captar mejor lo que ve, estamos hablando de un estilo visual. Si capta con mayor facilidad lo que oye, estamos hablando del canal auditivo, y si es más por lo que hace, nos referimos al canal kinestésico. Según los datos de este estudio y contrariamente a lo que algunos docentes y alumnos creen, entendemos que la utilización simultánea de más de uno de estos canales en una misma presentación ocasiona una carga cognitiva externa que entorpece la adquisición en L2, en lugar de fortalecerla.

\section{ALGUNAS IMPLICANCIAS METODOLÓGICAS}

Esta investigación tiene algunas implicancias prácticas. Desde el punto de vista pedagógico, el presente estudio sugiere que los docentes y diseñadores de material deben esforzarse por reducir la carga cognitiva innecesaria o externa; una realidad que eventualmente realzará la construcción de esquemas y automatización, procesos que facilitan el aprendizaje.

Existen medidas que favorecen el aprendizaje en la clase de lectura comprensiva de lengua extranjera. En primera instancia, los docentes deben reconsiderar el impacto de RE en la lectura de instrucciones en voz alta. Es una creencia popular compartida, pero desacertada, entre docentes de inglés que la lectura de instrucciones en voz alta junto con los alumnos facilita la comprensión lectora.

Otra práctica muy frecuente, aunque un tanto cuestionable porque genera el ER, es la lectura que el docente realiza en voz alta de un texto junto con sus alumnos, quienes luego han de explicar con sus propias palabras lo que han comprendido. En otros casos, los docentes suelen elegir a un alumno para que lea un texto, mientras que el resto de la clase lo hace silenciosamente al unísono. 
Una vez realizada la lectura, generalmente, se solicita a uno de los alumnos que reconstruya el texto. Con mucha frecuencia, los docentes leen un texto en voz alta junto a sus alumnos con la intención de asistir en la comprensión del mismo. El RE también se manifiesta en algunos exámenes internacionales estandarizados como el TOEFL, en su versión en papel. En este examen, los candidatos deben leer y escuchar las instrucciones en el manual de examen simultáneamente. El propósito de esta modalidad múltiple es asegurar que todos los candidatos hayan comprendido las instrucciones para completar el examen.

\section{LIMITACIONES DEL ESTUDIO}

Esta investigación ha brindado resultados interesantes que confirman nuestra hipótesis inicial. No obstante, existen limitaciones que merecen ser destacadas. Para comenzar, el número de participantes fue reducido. De haber incluido más participantes, nuestras conclusiones contarían con mayor fuerza y habrían permitido realizar algunas otras generalizaciones.

Hubiera sido interesante contar con mayor número de alumnos entrevistados. Esta información hubiera permitido contar con más reflexiones y opiniones acerca de la utilidad de la aplicación de uno u otro tratamiento, hecho que hubiera robustecido el cruce de los datos numéricos y los de valor. Por falta de tiempo y disponibilidad de los alumnos, esta idea no se pudo materializar.

Resulta importante destacar que la encuesta, uno de los instrumentos utilizados para la recolección de datos, no fue piloteada antes de su utilización en el estudio. Una muestra piloto hubiera permitido detectar posibles falencias en el instrumento, y corregirlas oportunamente. Por ejemplo, los resultados divididos en la pregunta $\mathrm{n}^{\circ} 5$, bien podrían ser el resultado de fallas en el diseño de este instrumento.

Finalmente, solamente tres evaluadores, trabajando primero individualmente y luego en grupos, analizaron e interpretaron las producciones de los alumnos para identificar las ideas principales. Si bien la técnica de inter-evaluación es útil, porque promueve la homogeneidad entre el modo de trabajar de los evaluadores, la participación de más jueces en este proceso habría contribuido a reforzar la validez y confiabilidad de los hallazgos. Porque el análisis de las muestras requiere de un cierto entrenamiento específico, no se pudo contar con un número mayor de evaluadores. Se espera que estos comentarios sean de utilidad para la realización de futuras investigaciones con similares características a las esbozadas en este estudio.

\section{INVESTIGACIONES A FUTURO}

Hasta el presente pocos estudios empíricos han sido realizados en ELT (Enseñanza del Inglés) en los que la TCC y el ER hayan sido explorados en la clase de lectura comprensiva de una lengua extranjera. Motivados por la necesidad de obtener más resultados empíricos, se llevaron a cabo una serie de estudios similares con grupos de futuros docentes de la Universidad Nacional de Mar del Plata (Cfr. 
Tuero y Gomez Laich, 2012a; Tuero y Gomez Laich, 2012b). Los resultados de estos experimentos demostraron que el modo redundante tuvo un efecto negativo en la habilidad de la comprensión lectora en L2 de los alumnos participantes. En la actualidad, se están llevando a cabo otros dos proyectos en contextos similares. Uno de ellos investiga si la complejidad de un texto determinado, junto con un modo de instrucción múltiple afecta las habilidades lectoras de alumnos que preparan el TOEFL. Utilizando una metodología más cualitativa, la otra investigación busca explorar en profundidad las creencias y preferencias de los alumnos al completar actividades de lectura utilizando un modo de instrucción simple y múltiple.

\section{BIBLIOGRAFÍA}

BAKER, David (2010): PET Result. Oxford: Oxford University Press.

BARNETT, Marva (1988): Reading through context: How real and perceived strategy use affects L2 comprehension. Modern Language Journal, 72, 150160.

BRANTMEIER, Cindy (2002): Second language reading strategy research at the secondary and university levels: Variations, disparities and generalizability. The Reading Matrix, 2, (3), 1-14.

CHANDLER, Paul y SWELLER, John (1991): Cognitive load theory and the format of instruction. Cognition and Instruction, 8, 293-332.

COOPER, Graham (1998): Research into Cognitive Load Theory and Instructional Design at UNSW. School of Education Studies, The University of New South Wales, Sydney.

CRAIG, Scotty, GHOLSON, Barry y DRISCOLL, David (2002): Animated pedagogical agents in multimedia educational environments: Effects of agent properties, picture features, and redundancy. Journal of Educational Psychology, 94, 428-434.

DENZIN, Norman (1989). The Research Act: A Theoretical Introduction to Sociological Methods, (3a. ed.). Englewood Cliffs, N. J., Prentice-Hall.

FAYER, Joan y KRASINSKI, Emily (1987): Native and nonative judgments of intelligibility and irritation. Language Learning, 37: 313-136.

KALYUGA, Slava, CHANDLER, Paul y SWELLER, John (2004): When redundant ton-screen text in multi-media technical instruction can interfere with learning. Human Factors, 46, 567-581.

KALYUGA, Slava, CHANDLER, Paul y SWELLER, John (2000): Incorporating learner experience into the design of multimedia instruction. Journal of Educational Psychology, 92, 126-136.

KINTSCH, Walter (1988): The role of knowledge in discourse comprehension: a construction-integrated model. Psychol. Rev. 95, 163-182. 
KINTSCH, Walter (1998): Comprehension: A Paradigm for Cognition. Cambridge University Press, Cambridge.

LAM, Yvonne (2009): Applying cognitive linguistics to teaching the Spanish Prepositions por And para. Language Awareness, 18 (1): 2-18.

LIGHTBOWN, Patsy (2000): Classroom SLA research and second language acquisition. Applied linguistics, 21: 431-462.

LIGHTBOWN, Patsy, SPADA, Nina, y WHITE, Lydia (Eds.) (1993): The role of instruction in second language acquisition. [Thematic issue]. Studies in Second Language Acquisition, 15, 2.

LYNCH, Brian (1996): Language Program Evaluation. Cambridge: Cambridge University Press.

MAYER, Richard, HEISER, Julie y LONN, Steve (2001): Cognitive constraints on multimedia learning: When presenting more material results in less understanding. Journal of Educational Psychology, 93, 187-198.

McNAMARA, Danielle y MAGLIANO, Joseph (2009): "Towards a comprehensive model of comprehension", en ROSS, B. (Ed.), The Psychology of Learning and Motivation (pp. 297-384). Academic Press: New York.

MILLER, George (1956): The magical number seven, plus or minus two: Some limits on our capacity for processing information. Psychological Review, 63, 81-97.

MOSS, Jarrot, SCHUNN, Christian, SCHNEIDER, Walter, MCNAMARA, Danielle y VANLEHN, Kurt (2011): The neural correlates of strategic reading comprehension: Cognitive control and discourse comprehension. NeuroImage, $58,675-686$.

MUNRO, Murray y DERWING, Tracey (1999): Foreign accent, comprehensibility and intelligibility in the speech of second language learners. Language Learning, 49, (1): 285-310.

SWELLER, John (1994): Cognitive load theory, learning difficulty, and instructional design. Learning and Instruction, 4, 295-312.

SWELLER, John (1999): Instructional design in technical areas. Camberwell, Victoria: Australian Council for Educational Research.

SWELLER, John (2005): “The redundancy principle", en MAYER R. E. (Ed.), Cambridge handbook of multimedia learning (pp. 159-167). New York: Cambridge University Press.

SWELLER, John y CHANDLER, Paul (1994): Why some material is difficult to learn. Cognition and Instruction, 12, 185-233.

SWELLER, John (2003): "Evolution of human cognitive architecture", en ROSS, B. (Ed.), The Psychology of Learning and Motivation (pp. 215-266). San Diego, CA: Academic Press. 
SWELLER, John (2004): Instructional design consequences of an analogy between evolution by natural selection and human cognitive architecture. Instructional Science, 32, 9-31.

TROFIMOVICH, Pavel y ISAACS, Talia (2012): Disentangling accent from comprehensibility. Bilingualism: Language and Cognition, 15 (4): 905-916.

TUERO, Susana y GÓMEZ LAICH, María Pía (2012a): Análisis de los efectos de presentaciones en modalidades múltiples en cognición. XIII Congreso de Lingüistica Sociedad Argentina de Lingüistica IFDC-San Luis, 27-30 marzo, 2012. Potrero de los Funes, San Luis, Argentina.

TUERO, Susana, LUCHINI, Pedro y GÓMEZ LAICH, María Pía (2012b): "Sobrecarga cognitiva: Relación entre presentaciones múltiples en el proceso de lectura", en GARCÍA, A. (Ed.), Aproximaciones teóricas y empíricas a la lingüística cognitiva (pp. 335-345). Mar del Plata: Editorial Martín.

VARONIS, E. M., y GASS, Susan (1982): The comprehensibility of nonnative speech. Studies in Second Language Acquisition, 4: 114-136.

WHITE, Joanna y RANTA, Leila (2002): Examining the interface between metalinguistic task performance and oral production in second language. Language Awareness, 11 (4): 259-290.

ZWAAN, Rolf, LANGSTON, Mark y GRAESSER, Arthur (1995): The construction of situation models in narrative comprehension: an event-indexing model. Psychological Science, 6, 292-297.

YALI, Diao, CHANDLER, Paul y SWELLER, John (2007): The effect of written text on comprehension of spoken English as a foreign language. American Journal of Psychology, 120, 2, 237-261.

ZWAAN, Rolf y RADVANSKY, Gabriel (1998): Situation models in language comprehension and memory. Psychological Bulletin (USA), 123, 162-185.

ZWAAN, Rolf (1999): Situation models: the mental leap into imagined worlds. Current Directions in Psychological Science, 8, 15-18. 